\title{
Hidden conformal symmetry of extremal Kaluza-Klein black hole in four dimensions
}

\author{
Yong-Chang Huang, Fang-Fang Yuan \\ Institute of Theoretical Physics, Beijing University of Technology \\ Beijing 100124, China \\ E-mail: ychuang@bjut.edu.cn, ffyuan@emails.bjut.edu.cn
}

\begin{abstract}
We study the hidden conformal symmetry of four-dimensional extremal Kaluza-Klein black hole. The scalar Laplacian corresponding to the radial equation in the near-region is rewritten in terms of the $S L(2, \mathbb{R})$ quadratic Casimir. Using the first law of black hole thermodynamics, this symmetry enables us to obtain the conjugate charges for the CFT side. The real-time correlators are also found to agree with the CFT expectations.
\end{abstract}




\section{Contents}

1. Introduction 1

2. Hidden conformal symmetry 2

3. Microscopic description 5

4. Real-time correlator 8

5. Conclusions 10

A. Four-dimensional Kaluza-Klein black hole 10

\section{Introduction}

The Kerr/CFT correspondence is a powerful duality that relates rotating black holes to two-dimensional conformal field theory (2D CFT) [1]. Since this gives us a way to get access to more realistic black holes, in particular, the Kerr black hole, rather than supersymmetric ones, it has invigorated very extensive studies since the advent ${ }^{1}$. Based on Brown-Henneaux formalism [2], i.e., by studying the asymptotic symmetry group, the Kerr/CFT correspondence has been applied to many different cases.

An important issue that perplexed the Kerr/CFT for some time is the microscopic description of non-extremal black holes. Remarkably, Castro, Maloney and Strominger uncovered the hidden conformal symmetry of non-extremal Kerr black hole in [14]. In a specific limit that is called near-region, it was found that the scalar Laplacian corresponding to the wave equation could be rewritten as the quadratic Casimir of an $S L(2, \mathbb{R}) \times S L(2, \mathbb{R})$ algebra. This was fulfilled by introducing a set of "conformal coordinates" and defining some vector fields as the generators of the algebra. In contrast with the extremal case, the local conformal symmetry in [14] was in the solution space of wave equation. The aspects of entropy and absorption cross section have also been discussed there for the non-extremal Kerr black hole. This formalism is quite universal which could be applied to many kinds of black holes as indicated by subsequent works [15] - 39].

In particular, a new set of conformal coordinates was introduced for extremal black holes in $[29]^{2}$. The $S L(2, \mathbb{R})$ quadratic Casimir was also obtained. Except the applications in [29], this has been extended to other black holes [23, 34, 37, 39]. Another new aspect of the hidden conformal symmetry was discovered in the study of Kerr-Newman black hole

\footnotetext{
${ }^{1}$ For a partial list of recent develepments, see [3] - 13.

${ }^{2} \mathrm{~A}$ related work appeared in [28].
} 
[26]. The usual method based on [14] can be used to investigate the wave equation which involves three charges, i.e., mass $M$, angular momentum $J$, and charge $Q$. However, the observation of the authors was that when the quantum number of angular momentum $J$ was taken to zero and a new vector operator was introduced for the charge $Q$, another hidden conformal symmetry emerged, which was called Q-picture description. (In view of this, the former description was named as J-picture.) Certain black holes have been analyzed from this viewpoint [36, 38].

In this work, we will explore the hidden conformal symmetry of four-dimensional (4D) extremal Kaluza-Klein black hole using the set of conformal coordinates introduced in [29]. Note that the 4D non-extremal Kaluza-Klein black hole has been studied in [19] along the line with [14]. However, the extremal case is nontrivial as shown in previous works on other black holes.

The organization of the paper is as follows. In the next section, following [29], we reproduce the scalar Laplacian of the radial wave equation by deriving the quadratic Casimir, which is the essential indicator of the hidden conformal symmetry. In section 3, we relate the first law of black hole thermodynamics to the CFT expression of entropy, and obtain the conjugate charges. In section 1 , the Euclidean correlator is written down and we compare it with the real-time correlator on the gravity side. Other microscopic aspects of the duality are also discussed in these two sections. In particular, besides the extremal case, we determine the form of conjugate charges and real-time correlator for the non-extremal case as complementary to the previous study [19]. The conclusions and remarks are included in section 5 .

\section{Hidden conformal symmetry}

In this section, we will adapt the conformal coordinates of [29] to 4D extremal Kaluza-Klein black hole, and reproduce the scalar wave equation in near-region in the low frequency limit. Some basic formulas of Kaluza-Klein black hole can be found in Appendix A.

We start with the Klein-Gordon equation for a massless scalar field in the background of 4D Kaluza-Klein black hole

$$
\frac{1}{\sqrt{-g}} \partial_{\mu}\left(\sqrt{-g} g^{\mu \nu} \partial_{\nu}\right) \Phi=0
$$

By using the ansatz of the wave function as

$$
\Phi=e^{-i \omega t+i m \varphi} \Phi(r, \theta)
$$

and taking a near-region limit which is dictated by

$$
\omega \mu \ll 1, \quad \omega r \ll 1,
$$

we obtain the reduced radial wave equation 19

$$
\begin{aligned}
& {\left[\partial_{r} \Delta \partial_{r}+\frac{\left(2 \mu r_{+} \omega / \sqrt{1-\nu^{2}}-a m\right)^{2}}{\left(r-r_{+}\right)\left(r_{+}-r_{-}\right)}-\frac{\left(2 \mu r_{-} \omega / \sqrt{1-\nu^{2}}-a m\right)^{2}}{\left(r-r_{-}\right)\left(r_{+}-r_{-}\right)}\right] R(r)} \\
& =l(l+1) R(r) .
\end{aligned}
$$


As we will concentrate on the extremal limit $\mu=a$, the equation simplifies further as

$$
\begin{aligned}
& {\left[\partial_{r} \Delta \partial_{r}+\frac{2\left(2 \mu \omega / \sqrt{1-\nu^{2}}\right)\left(2 \mu r_{+} \omega / \sqrt{1-\nu^{2}}-a m\right)}{r-r_{+}}+\frac{\left(2 \mu r_{+} \omega / \sqrt{1-\nu^{2}}-a m\right)^{2}}{\left(r-r_{+}\right)^{2}}\right] R(r)} \\
& =l(l+1) R(r) \text {. }
\end{aligned}
$$

The conformal coordinates for extremal black holes introduced in 29] are

$$
\begin{aligned}
\omega^{+} & =\frac{1}{2}\left(\alpha_{1} t+\beta_{1} \varphi-\frac{\gamma_{1}}{r-r_{+}}\right), \\
\omega^{-} & =\frac{1}{2}\left(\exp \left(2 \pi T_{L} \varphi+2 n_{L} t\right)-\frac{2}{\gamma_{1}}\right), \\
y & =\sqrt{\frac{\gamma_{1}}{2\left(r-r_{+}\right)}} \exp \left(\pi T_{L} \varphi+n_{L} t\right) .
\end{aligned}
$$

Thus the explicit expression for the quadratic Casimir is

$$
\begin{array}{r}
H^{2}=\partial_{r}\left(\Delta \partial_{r}\right)-\left(\frac{\gamma_{1}\left(2 \pi T_{L} \partial_{t}-2 n_{L} \partial_{\varphi}\right)}{A\left(r-r_{+}\right)}\right)^{2} \\
-\frac{2 \gamma_{1}\left(2 \pi T_{L} \partial_{t}-2 n_{L} \partial_{\varphi}\right)}{A^{2}\left(r-r_{+}\right)}\left(\beta_{1} \partial_{t}-\alpha_{1} \partial_{\varphi}\right)
\end{array}
$$

where $A=2 \pi T_{L} \alpha_{1}-2 n_{L} \beta_{1}$, and $\Delta=\left(r-r_{+}\right)^{2}$. Through the comparison of equations (2.5) and (2.9), we have the identifications

$$
\alpha_{1}=0, \quad \beta_{1}=-\frac{\gamma_{1}}{\mu}, \quad 2 \pi T_{L}=1, \quad n_{L}=\frac{\sqrt{1-\nu^{2}}}{4 \mu},
$$

and also $A=\frac{\sqrt{1-\nu^{2}}}{2 \mu^{2}} \gamma_{1}$. Note that although the equation about $T_{L}$ here is obtained by a detailed comparison, it is the same as that of [19] where a derivation was given. The fact that the radial wave equation (2.5) could be reproduced by the quadratic Casimir in (2.9) in some sense justifies the universal applicability of the new set of conformal coordinates proposed in [29].

Following the logic of [14], we demonstrate the hidden conformal symmetry of 4D extremal Kaluza-Klein black hole explicitly as below.

Firstly, we introduce the conformal coordinates

$$
\begin{aligned}
\omega^{+} & =-\frac{\gamma_{1}}{2}\left(\frac{\varphi}{\mu}+\frac{1}{r-\mu}\right), \\
\omega^{-} & =\frac{1}{2}\left(\exp \left(\varphi+\frac{\sqrt{1-\nu^{2}}}{2 \mu} t\right)-\frac{2}{\gamma_{1}}\right), \\
y & =\sqrt{\frac{\gamma_{1}}{2(r-\mu)}} \exp \left(\frac{\varphi}{2}+\frac{\sqrt{1-\nu^{2}}}{4 \mu} t\right) .
\end{aligned}
$$


Then we define locally the vector fields

$$
\begin{aligned}
H_{1} & =i \partial_{+} \\
& =i \frac{2 \mu}{\gamma_{1}}\left(\frac{2 \mu}{\sqrt{1-\nu^{2}}} \partial t-\partial \varphi\right) \\
H_{0} & =i\left(\omega^{+} \partial_{+}+\frac{1}{2} y \partial_{y}\right) \\
& =-i\left[(r-\mu) \partial r+\frac{2 \mu}{\sqrt{1-\nu^{2}}} \partial t-\partial \varphi\right] \\
H_{-1} & =i\left(\omega^{+2} \partial_{+}+\omega^{+} y \partial_{y}-y^{2} \partial_{-}\right) \\
& =i \gamma_{1}\left[\frac{r-\mu}{\mu} \varphi \partial r+\frac{1}{\sqrt{1-\nu^{2}}}\left(\varphi^{2}+\frac{\mu(3 \mu-2 r)}{(r-\mu)^{2}}\right) \partial t-\frac{1}{2 \mu}\left(\varphi^{2}+\frac{\mu^{2}}{(r-\mu)^{2}}\right) \partial \varphi\right]
\end{aligned}
$$

and

$$
\begin{aligned}
\bar{H}_{1} & =i \partial_{-} \\
& =i \frac{2}{r-\mu} \exp \left(-\varphi-\frac{\sqrt{1-\nu^{2}}}{2 \mu} t\right)\left[(r-\mu)^{2} \partial r+\mu\left(\frac{2(r-2 \mu)}{\sqrt{1-\nu^{2}}} \partial t+\partial \varphi\right)\right] \\
\bar{H}_{0} & =i\left(\omega^{-} \partial_{-}+\frac{1}{2} y \partial_{y}\right) \\
& =-i \frac{2}{\gamma_{1}(r-\mu)} \exp \left(-\varphi-\frac{\sqrt{1-\nu^{2}}}{2 \mu} t\right)\left[(r-\mu)^{2} \partial r+\mu\left(\frac{2(r-2 \mu)}{\sqrt{1-\nu^{2}}} \partial t+\partial \varphi\right)\right]+i \frac{2 \mu}{\sqrt{1-\nu^{2}}} \partial t \\
\bar{H}_{-1} & =i\left(\omega^{-2} \partial_{-}+\omega^{-} y \partial_{y}-y^{2} \partial_{+}\right) \\
& =-i \frac{1}{2(r-\mu)} \exp \left(\varphi+\frac{\sqrt{1-\nu^{2}}}{2 \mu} t\right)\left[(r-\mu)^{2} \partial r-\mu\left(\frac{2(r-2 \mu)}{\sqrt{1-\nu^{2}}} \partial t-\partial \varphi\right)\right] \\
& +i \frac{2}{\gamma_{1}^{2}(r-\mu)} \exp \left(-\varphi-\frac{\sqrt{1-\nu^{2}}}{2 \mu} t\right)\left[(r-\mu)^{2} \partial r+\mu\left(\frac{2(r-2 \mu)}{\sqrt{1-\nu^{2}}} \partial t+\partial \varphi\right)\right]-i \frac{4 \mu}{\gamma_{1} \sqrt{1-\nu^{2}}} \partial t
\end{aligned}
$$

each set of which satisfies the $S L(2, \mathbb{R})$ algebra

$$
\begin{array}{rlrl}
{\left[H_{0}, H_{ \pm 1}\right]} & =\mp i H_{ \pm 1}, & & {\left[H_{-1}, H_{1}\right]=-2 i H_{0},} \\
{\left[\bar{H}_{0}, \bar{H}_{ \pm 1}\right]=\mp i \bar{H}_{ \pm 1},} & {\left[\bar{H}_{-1}, \bar{H}_{1}\right]=-2 i \bar{H}_{0} .}
\end{array}
$$

In contrast with the extremal case, we have a free parameter $\gamma_{1}$ here.

The quadratic Casimir is

$$
H^{2}=\bar{H}^{2}=-H_{0}^{2}+\frac{1}{2}\left(H_{1} H_{-1}+H_{-1} H_{1}\right)=\frac{1}{4}\left(y^{2} \partial_{y}^{2}-y \partial_{y}\right)+y^{2} \partial_{+} \partial_{-} .
$$

By inserting equations (2.14) - (2.19) into the formula (2.22), we find that the expression of the Casimir is exactly that of the scalar Laplacian in (2.5).

Some comments about the locality of the vector fields defined in equations (2.17) (2.19) are in order. It can be attributed to the fact that under the periodic identification 
of the angular coordinate $\varphi \sim \varphi+2 \pi$, they do not behave periodically ${ }^{3}$. Since they are not globally defined, we cannot use them to generate new global solutions. The corresponding behaviors of conformal coordinates given in equations (2.11) - (2.13) can also be shown explicitly as follows

$$
\omega^{+} \sim \omega^{+}-\pi \frac{\gamma_{1}}{\mu}, \quad \omega^{-} \sim e^{2 \pi}\left(\omega^{-}+\frac{1}{\gamma_{1}}\right)-\frac{1}{\gamma_{1}}, \quad y \sim e^{\pi} y
$$

where the tranformation property of $\omega^{+}$is distinct from that of $\omega^{-}$and $y$. This identification is generated by the group element

$$
e^{-i 2 \pi \bar{H}_{0}}
$$

Accordingly, one factor of the $S L(2, \mathbb{R})_{L} \times S L(2, \mathbb{R})_{R}$ symmetry is spontaneously broken to the $U(1)$ subgroup.

Through the comparison between $(2.23)$ and the transformation behaviors of conformal coordinates in non-extremal [14] or extremal Kerr/CFT [29], one may read off the temperature as $T_{L}=\frac{1}{2 \pi}$. It also justifies that only one sector exists for the $4 \mathrm{D}$ extremal Kaluza-Klein black hole. On the other hand, from the relation

$$
\omega^{-} \sim e^{-t^{-}}
$$

where $t^{-}=-\varphi-\frac{\sqrt{1-\nu^{2}}}{2 \mu}$, one finds that an observer at fixed position in the Rindler coordinate $t^{-}$will observe a thermal bath of Unruh radiation with temperature $T_{L}=\frac{1}{2 \pi}$.

The hidden conformal symmetry is peculiar in that it pertains to the solution space of near-region wave equation rather than the geometry. It is exactly this broken $S L(2, \mathbb{R})$ symmetry that enables us to investigate other aspects of the correspondence in the next two sections ${ }^{4}$.

\section{Microscopic description}

To be complete, we start with the non-extremal story. The microsopic entropy of $4 \mathrm{D}$ Kaluza-Klein black hole is [19]

$$
S=\frac{\pi^{2}}{3}\left(c_{L} T_{L}+c_{R} T_{R}\right)=2 \pi \frac{\mu}{\sqrt{1-\nu^{2}}}\left(\mu+\sqrt{\mu^{2}-a^{2}}\right)
$$

where

$$
c_{L}=c_{R}=\frac{12 a \mu}{\sqrt{1-\nu^{2}}}, \quad T_{L}=\frac{r_{+}+r_{-}}{4 \pi a}, \quad T_{R}=\frac{r_{+}-r_{-}}{4 \pi a} .
$$

This agrees with the macrocopic Bekenstein-Hawking area law.

\footnotetext{
${ }^{3}$ This argument associated with rotating black holes was originally given in 14 in the context of nonextremal Kerr/CFT.

${ }^{4}$ Note that the essence of the original (extremal) Kerr/CFT [1] is the realization of Virasoro algebra from an enhancement of the rotational $U(1)$ isometry. It also relies on Brown-Henneaux formalism [2].
} 
In what follows, we determine the conjugate charges from the first law of thermodynamics. On the CFT side, the entropy is

$$
\delta S=\frac{\delta E_{L}}{T_{L}}+\frac{\delta E_{R}}{T_{R}}
$$

while on the black hole side, we have

$$
\delta S=\frac{1}{T_{H}} \delta M-\frac{\Phi_{H}}{T_{H}} \delta Q-\frac{\Omega_{H}}{T_{H}} \delta J
$$

which is the first law. By comparing these two equations, we get the conjugate charges 18

$$
\begin{aligned}
& \delta E_{L}=\frac{\left(2 M^{2}-Q^{2}\right) M}{J} \delta M-\left(\frac{M^{2} Q}{J}-\frac{Q^{3}}{2 J}\right) \delta Q, \\
& \delta E_{R}=\frac{\left(2 M^{2}-Q^{2}\right) M}{J} \delta M-\frac{M^{2} Q}{J} \delta Q-\delta J .
\end{aligned}
$$

However, as for our specific case, it is convenient and necessary to express them via the parameters $\mu, \nu$, and $a$, instead of the physical parameters $M, Q$, and $J$. Note that the discussions concerning the hidden conformal symmetry of 4D Kaluza-Klein black hole as in the above section and in [19] all use these parameters.

Based on the formulas in Appendix A, the variations of $M, Q$, and $J$ lead to

$$
\begin{array}{r}
\delta M=\frac{1}{1-\nu^{2}}\left(\frac{2-\nu^{2}}{2} \delta \mu+\frac{\mu \nu}{1-\nu^{2}} \delta \nu\right), \\
\delta Q=\frac{1}{1-\nu^{2}}\left(\nu \delta \mu+\mu \frac{1+\nu^{2}}{1-\nu^{2}} \delta \nu\right), \\
\delta J=\frac{1}{\sqrt{1-\nu^{2}}}\left(a \delta \mu+\frac{a \mu \nu}{1-\nu^{2}} \delta \nu+\mu \delta a\right) .
\end{array}
$$

Using the expressions of angular velocity and electric potential in A.8, we obtain

$$
T_{H} \delta S=\frac{r_{+}^{2}}{r_{+}^{2}+a^{2}} \delta \mu+\frac{\nu}{2\left(1-\nu^{2}\right)} \sqrt{\mu^{2}-a^{2}} \delta \nu-\frac{a \mu}{r_{+}^{2}+a^{2}} \delta a .
$$

Recall that $T_{H}=\frac{\sqrt{1-\nu^{2}}}{2 \pi} \frac{\sqrt{\mu^{2}-a^{2}}}{r_{+}^{2}+a^{2}}$, so the variation of entropy can be written as

$$
\delta S=\frac{\pi}{\sqrt{1-\nu^{2}}}\left(\frac{2 r_{+}^{2}}{\sqrt{\mu^{2}-a^{2}}} \delta \mu+\frac{\nu}{1-\nu^{2}}\left(r_{+}^{2}+a^{2}\right) \delta \nu-\frac{2 a \mu}{\sqrt{\mu^{2}-a^{2}}} \delta a\right) .
$$

Then via the comparison of this equation and the CFT fromula (3.3), and with the help of (3.2), finally the conjugate charges read as

$$
\begin{aligned}
\delta E_{L} & =\frac{2 \mu}{\sqrt{1-\nu^{2}}} \delta \mu+\frac{\mu^{2} \nu}{\left(1-\nu^{2}\right)^{3 / 2}} \delta \nu \\
\delta E_{R} & =\frac{r_{+}^{2}-2 a \sqrt{\mu^{2}-a^{2}}}{a \sqrt{1-\nu^{2}}} \delta \mu+\frac{\mu \nu \sqrt{\mu^{2}-a^{2}}}{a\left(1-\nu^{2}\right)^{3 / 2}}\left(r_{+}-a\right) \delta \nu-\frac{\mu}{\sqrt{1-\nu^{2}}} \delta a .
\end{aligned}
$$


With the identifications

$$
\begin{aligned}
& \delta \mu=\omega, \quad \delta \nu=e, \quad \delta a=m \\
& \omega_{L}=\frac{2 \mu}{\sqrt{1-\nu^{2}}} \omega, \quad \omega_{R}=\frac{r_{+}^{2}-2 a \sqrt{\mu^{2}-a^{2}}}{a \sqrt{1-\nu^{2}}} \omega-\frac{\mu}{\sqrt{1-\nu^{2}}} m \\
& q_{L}=q_{R}=\delta \nu=e, \\
& \mu_{L}=-\frac{\mu^{2} \nu}{\left(1-\nu^{2}\right)^{3 / 2}}, \quad \mu_{R}=-\frac{\mu \nu \sqrt{\mu^{2}-a^{2}}}{a\left(1-\nu^{2}\right)^{3 / 2}}\left(r_{+}-a\right)
\end{aligned}
$$

we have

$$
\delta E_{L}=\omega_{L}-q_{L} \mu_{L}, \quad \delta E_{R}=\omega_{R}-q_{R} \mu_{R}
$$

Then we turn to the extremal case, and the quantities corresponding to the extremal case will be denoted with a prime. Now the microscopic entropy comes entirely from the left sector and takes the form

$$
S^{\prime}=\frac{\pi^{2}}{3} c_{L} T_{L}^{\prime}=2 \pi \frac{\mu^{2}}{\sqrt{1-\nu^{2}}}
$$

where $T_{L}^{\prime}=\frac{1}{2 \pi}$.

Note that the CFT formula (3.3) is now modified to

$$
\delta S^{\prime}=\frac{\delta E_{L}^{\prime}}{T_{L}^{\prime}} .
$$

Proceeding as the above discussion, the variation of the extremal entropy is

$$
\delta S^{\prime}=\frac{4 \pi \mu}{\sqrt{1-\nu^{2}}} \delta \mu+\frac{2 \pi \mu^{2} \nu}{\left(1-\nu^{2}\right)^{3 / 2}} \delta \nu .
$$

Thus the conjugate charge is immediately found as

$$
\delta E_{L}^{\prime}=\frac{2 \mu}{\sqrt{1-\nu^{2}}} \delta \mu+\frac{\mu^{2} \nu}{\left(1-\nu^{2}\right)^{3 / 2}} \delta \nu
$$

If we identify

$$
\begin{aligned}
\delta \mu & =\omega, \quad \delta \nu=e, \quad \delta a=m \\
\omega_{L}^{\prime} & =\frac{2 \mu}{\sqrt{1-\nu^{2}}} \omega \\
q_{L}^{\prime} & =\delta \nu=e \\
\mu_{L}^{\prime} & =-\frac{\mu^{2} \nu}{\left(1-\nu^{2}\right)^{3 / 2}},
\end{aligned}
$$

it follows that

$$
\delta E_{L}^{\prime}=\omega_{L}^{\prime}-q_{L}^{\prime} \mu_{L}^{\prime}
$$

Note that the identifications $(3.22)-(3.25)$ are the same as the left sector part of those for the non-extremal case (3.13) - (3.16).

To sum up, we have derived the conjugate charges for both the $4 \mathrm{D}$ non-extremal and extremal Kaluza-Klein black holes. 


\section{Real-time correlator}

In this section, we will compare the Euclidean correlator on the CFT side to the real-time correlator on the black hole side, and find the agreement. A general study of the real-time correlators in the Kerr/CFT correspondence can be found in 40. For the discussion about the extremal case, see [29].

In a $2 \mathrm{D}$ CFT, the two-point functions of the primary operators are determined by the conformal invariance. The Euclidean correlator is related to the retarded correlator as

$$
G_{E}\left(\omega_{L, E}, \omega_{R, E}\right)=G_{R}\left(i \omega_{L, E}, i \omega_{R, E}\right), \quad \omega_{L, E}, \omega_{R, E}>0 .
$$

At finite temperature, $\omega_{L, E}$ and $\omega_{R, E}$ take discrete values of the Matsubara frequencies

$$
\omega_{L, E}=2 \pi m_{L} T_{L}, \quad \omega_{R, E}=2 \pi m_{R} T_{R}
$$

For an operator of dimensions $\left(h_{L}, h_{R}\right)$, the momentum space Euclidean correlator is given by 41]

$$
\begin{aligned}
G_{E}\left(\omega_{L, E}, \omega_{R, E}\right) \sim & T_{L}^{2 h_{L}-1} T_{R}^{2 h_{R}-1} e^{i \frac{\bar{\omega}_{L, E}}{2 T_{L}}} e^{i \frac{\bar{\omega}_{R, E}}{2 T_{R}}} \\
& \cdot \Gamma\left(h_{L}+\frac{\bar{\omega}_{L, E}}{2 \pi T_{L}}\right) \Gamma\left(h_{L}-\frac{\bar{\omega}_{L, E}}{2 \pi T_{L}}\right) \Gamma\left(h_{R}+\frac{\bar{\omega}_{R, E}}{2 \pi T_{R}}\right) \Gamma\left(h_{R}-\frac{\bar{\omega}_{R, E}}{2 \pi T_{R}}\right),
\end{aligned}
$$

where

$$
\bar{\omega}_{L, E}=\omega_{L, E}-i q_{L} \mu_{L}, \quad \bar{\omega}_{R, E}=\omega_{R, E}-i q_{R} \mu_{R} .
$$

On the gravity side, for a scalar field in a black hole background, the prescription for two-point real-time correlators was originally proposed in [42]. We will follow [40] where this technique has been adapted to the Kerr black hole.

For the 4D Kaluza-Klein black hole, by introducing

$$
z=\frac{r-r_{+}}{r-r_{-}}
$$

the radial equation (2.4) can be written as

$$
(1-z) z \partial_{z}^{2} R(z)+(1-z) \partial_{z} R(z)+\left(\frac{A_{1}}{z}+A_{2}+\frac{A_{3}}{1-z}\right) R(z)=0
$$

where

$$
\begin{aligned}
& A_{1}=\frac{\left(2 \mu r_{+} \omega / \sqrt{1-\nu^{2}}-a m\right)^{2}}{\left(r_{+}-r_{-}\right)^{2}}, \\
& A_{2}=-\frac{\left(2 \mu r_{-} \omega / \sqrt{1-\nu^{2}}-a m\right)^{2}}{\left(r_{+}-r_{-}\right)^{2}}, \\
& A_{3}=-l(l+1) .
\end{aligned}
$$

The central information comes from the asymptotic behavior

$$
R(r) \sim A r^{h-1}+B r^{-h}
$$


where $h$ is the conformal weight

$$
h=l+1 .
$$

The retarded Green's function is then

$$
G_{R} \sim \frac{\Gamma(1-2 h)}{\Gamma(2 h-1)} \frac{\Gamma\left(h+i \frac{\bar{\omega}_{L}}{2 \pi T_{L}}\right) \Gamma\left(h+i \frac{\bar{\omega}_{R}}{2 \pi T_{R}}\right)}{\Gamma\left(1-h+i \frac{\bar{\omega}_{L}}{2 \pi T_{L}}\right) \Gamma\left(1-h+i \frac{\bar{\omega}_{R}}{2 \pi T_{R}}\right)} .
$$

With the identifications $(3.13)-(3.16)$, the Green's function is obviously in agreement with the CFT expression (4.3).

For the extremal case, only the left sector contributes. However, as noted in [29], the asymptotic behavior is nontrivially different from the above discussion as indicated below. As in the above section, the extremal quantities will be denoted with a prime to distinguish from the non-extremal ones.

On the CFT side, the momentum space Euclidean correlator is now given by

$$
G_{E}\left(\omega_{L, E}^{\prime}, \omega_{R, E}^{\prime}\right) \sim T_{L}^{\prime 2 h_{L}^{\prime}-1} e^{i \frac{\bar{\omega}_{L, E}^{\prime}}{2 T_{L}^{\prime}}} \Gamma\left(h_{L}^{\prime}+\frac{\bar{\omega}_{L, E}^{\prime}}{2 \pi T_{L}^{\prime}}\right) \Gamma\left(h_{L}^{\prime}-\frac{\bar{\omega}_{L, E}^{\prime}}{2 \pi T_{L}^{\prime}}\right),
$$

where

$$
\bar{\omega}_{L, E}^{\prime}=\omega_{L, E}^{\prime}-i q_{L}^{\prime} \mu_{L}^{\prime} .
$$

The extremal radial equation (2.5) can be written as

$$
\left[\partial_{r} \Delta \partial_{r}+\frac{C}{r-r_{+}}+\frac{D^{2}}{\left(r-r_{+}\right)^{2}}\right] R(r)=l(l+1) R(r)
$$

where

$$
\begin{aligned}
& C=2\left(2 \mu \omega / \sqrt{1-\nu^{2}}\right)\left(2 \mu r_{+} \omega / \sqrt{1-\nu^{2}}-a m\right) \\
& D=2 \mu r_{+} \omega / \sqrt{1-\nu^{2}}-a m .
\end{aligned}
$$

Introduce $x=\frac{-2 i D}{r-r_{+}}$, then the equation leads to

$$
\frac{d^{2} R}{d x^{2}}+\left(\frac{\frac{1}{4}-m_{s}^{2}}{x^{2}}+\frac{k}{2}-\frac{1}{4}\right) R(x)=0
$$

where

$$
k=i 2 \mu \omega / \sqrt{1-\nu^{2}}, \quad m_{s}^{2}=\frac{1}{4}+l(l+1) .
$$

Different from the non-extremal case (4.8), the asymptotic behavior is now

$$
R \sim C_{1} r^{-h^{\prime}}+C_{2} r^{1-h^{\prime}}
$$

where $h$ is the conformal weight

$$
h^{\prime}=\frac{1}{2}+m_{s}=\frac{1}{2}+\sqrt{\frac{1}{4}+l(l+1)} .
$$

Thus the retarded Green function is

$$
G_{R} \sim \frac{C_{1}}{C_{2}} \propto \frac{\Gamma\left(1-2 h^{\prime}\right) \Gamma\left(h^{\prime}-k\right)}{\Gamma\left(2 h^{\prime}-1\right) \Gamma\left(1-h^{\prime}-k\right)} .
$$

With the identifications (3.22) - (3.25) for the 4D extremal Kaluza-Klein black hole, we find the agreement with the Euclidean correlator on the CFT side (4.11). 


\section{Conclusions}

It has been shown in [29] that in contrast with the non-extremal case, to study the hidden conformal symmetry of extremal black holes, a new set of conformal coordinates should be introduced. In this paper, we extended this to study the 4D extremal Kaluza-Klein black hole. The scalar Laplacian corresponding to the radial equation in the near-region can be rewritten in terms of the $S L(2, \mathbb{R})$ quadratic Casimir. Based on the relation between the first law of black hole thermodynamics and the CFT expression of entropy, we have obtained the conjugate charges for both the 4D non-extremal and extremal Kaluza-Klein black holes. The real-time correlators also agreed with the CFT expressions. Obviously, the fermionic and vector cases of the scattering and real-time correlators can be investigated analogously which we omitted here.

Some possible extentions of this work are as follows. Firstly, it may be interesting to find a relation with the holographic Q-picture description proposed in [26], whose main point has been sketched in the introduction. Just as the Kerr-Newman black hole, the 4D Kaluza-Klein black hole has three kinds of charges. However, all the discussions here used the new parameters $(\mu, \nu$, and $a)$ instead of the physical parameters $(M, Q$, and $J)$. The procedure introduced in [26], especially taking the limit of the wave equation, may need to be slightly modified for our specific case. Secondly, the hidden conformal symmetry of 5D rotating Kaluza-Klein black hole [43, 44] may deserve to study. In particular, the results of the extremal case should be consistent with those in [45], where the asymptotic symmetry group of this black hole was explored. Finally, a physical explanation may be found for the free parameter in conformal coordinates which exists for the extremal black holes ${ }^{5}$. One may anticipate its relation with the special properties of near horizon geometry.

Although the discovery of hidden conformal symmetry is a breakthrough for the nonextremal Kerr/CFT correspondence, a more systematic approach is still expected. Other than the continuous appearance of new ideas, many corners and aspects of the whole picture remain to be explored.

\section{Acknowledgments}

This work was supported by National Natural Science Foundation of China (No. 10875009) and by Beijing Natural Science Foundation (No. 1072005).

\section{A. Four-dimensional Kaluza-Klein black hole}

We review the basics of four-dimensional Kaluza-Klein black hole in this appendix. The Kaluza-Klein black hole is an exact solution of the following Einstein-Maxwell-dilaton action with $\alpha=\sqrt{3}$

$$
S=\frac{1}{16 \pi} \int d^{4} x \sqrt{-g}\left[R-2(\nabla \phi)^{2}-e^{-2 \alpha \phi} F^{2}\right] .
$$

\footnotetext{
${ }^{5}$ See equations $(2.6)-(2.8)$. Note that $\gamma_{1}$ is a free parameter which is absent in the non-extremal case.
} 
It can be obtained from dimensional reduction of the boosted five-dimensional Kerr solution to four dimensions. The metric is 46] - 48

$$
\begin{aligned}
d s^{2}= & -\frac{\Delta-a^{2} \sin ^{2} \theta}{B \Sigma} d t^{2}-2 a \sin ^{2} \theta \frac{Z}{B \sqrt{1-\nu^{2}}} d t d \varphi \\
& +\left[B\left(r^{2}+a^{2}\right)+a^{2} \sin ^{2} \theta \frac{Z}{B}\right] \sin ^{2} \theta d \varphi^{2} \\
& +\frac{B \Sigma}{\Delta} d r^{2}+B \Sigma d \theta^{2},
\end{aligned}
$$

where

$$
\Delta=r^{2}-2 \mu r+a^{2}, \quad \Sigma=r^{2}+a^{2} \cos ^{2} \theta, \quad Z=\frac{2 \mu r}{\Sigma}, \quad B=\sqrt{1+\frac{\nu^{2} Z}{1-\nu^{2}}} .
$$

The dilaton field is $\phi=-(\sqrt{3} / 2) \ln B$, and the components of gauge potential are

$$
A_{t}=\frac{\nu Z}{2\left(1-\nu^{2}\right) B^{2}}, \quad A_{\varphi}=-\frac{a \nu Z \sin ^{2} \theta}{2 \sqrt{1-\nu^{2}} B^{2}} .
$$

The physical mass $M$, the charge $Q$, and the angular momentum $J$ are expressed through the mass parameter $\mu$, boost parameter $\nu$, and specific angular momentum $a$ as follows

$$
\begin{aligned}
& M=\mu\left[1+\frac{\nu^{2}}{2\left(1-\nu^{2}\right)}\right], \\
& Q=\frac{\mu \nu}{1-\nu^{2}}, \quad J=\frac{\mu a}{\sqrt{1-\nu^{2}}},
\end{aligned}
$$

and the locations of the horizons are at

$$
r_{ \pm}=\mu \pm \sqrt{\mu^{2}-a^{2}}
$$

The angular velocity and electric potential of the event horizon are, respectively, given by

$$
\Omega_{H}=\frac{a \sqrt{1-\nu^{2}}}{r_{+}^{2}+a^{2}}, \quad \Phi_{H}=\frac{Q r_{+}\left(1-\nu^{2}\right)}{r_{+}^{2}+a^{2}}=\frac{\nu}{2} .
$$

Hawking temperature and entropy of the black hole are

$$
\begin{aligned}
T_{H} & =\frac{\sqrt{1-\nu^{2}}}{2 \pi} \frac{\sqrt{\mu^{2}-a^{2}}}{r_{+}^{2}+a^{2}}, \\
S & =2 \pi \frac{\mu}{\sqrt{1-\nu^{2}}}\left(\mu+\sqrt{\mu^{2}-a^{2}}\right) .
\end{aligned}
$$

In this work, we will focus on the extremal limit $\mu=a$ when the entropy is

$$
S\left(T_{H}=0\right)=\frac{2 \pi \mu^{2}}{\sqrt{1-\nu^{2}}}
$$




\section{References}

[1] M. Guica, T. Hartman, W. Song and A. Strominger, "The Kerr/CFT Correspondence," Phys. Rev. D 80 (2009) 124008 [arXiv:0809.4266 [hep-th]].

[2] J. D. Brown and M. Henneaux, "Central Charges in the Canonical Realization of Asymptotic Symmetries: An Example from Three-Dimensional Gravity," Commun. Math. Phys. 104 (1986) 207.

[3] J. de Boer, M. M. Sheikh-Jabbari and J. Simon, "Near Horizon Limits of Massless BTZ and Their CFT Duals," [arXiv:1011.1897 [hep-th]].

[4] V. Balasubramanian, J. Parsons and S. F. Ross, "States of a chiral 2d CFT," [arXiv:1011.1803 [hep-th]].

[5] Y. Matsuo and T. Nishioka, "New Near Horizon Limit in Kerr/CFT," [arXiv:1010.4549 [hep-th]].

[6] T. Azeyanagi, N. Ogawa and S. Terashima, "Emergent $\mathrm{AdS}_{3}$ in the Zero Entropy Extremal Black Holes," [arXiv:1010.4291 [hep-th]].

[7] J. Gegenberg, H. Liu, S. S. Seahra and B. K. Tippett, "A Tomimatsu-Sato/CFT correspondence," [arXiv:1010.2803 [hep-th]].

[8] M. Guica and A. Strominger, "Microscopic Realization of the Kerr/CFT Correspondence," [arXiv:1009.5039 [hep-th]].

[9] A. Galajinsky, "Particle dynamics near extreme Kerr throat and supersymmetry," JHEP 1011 (2010) 126 [arXiv:1009.2341 [hep-th]].

[10] B. K. Button, L. Rodriguez and C. A. Whiting, "A Near Horizon CFT Dual for Kerr-Newman-AdS," [arXiv:1009.1661 [hep-th]].

[11] S. Detournay, D. Israel, J. M. Lapan and M. Romo, "String Theory on Warped $\mathrm{AdS}_{3}$ and Virasoro Resonances," [arXiv:1007.2781 [hep-th]].

[12] A. P. Porfyriadis and F. Wilczek, "Effective Action, Boundary Conditions, and Virasoro Algebra for $\mathrm{AdS}_{3}, "$ [arXiv:1007.1031 [gr-qc]].

[13] B. C. da Cunha and A. R. de Queiroz, "Kerr-CFT From Black-Hole Thermodynamics," JHEP 1008 (2010) 076 [arXiv:1006.0510 [hep-th]].

[14] A. Castro, A. Maloney and A. Strominger, "Hidden Conformal Symmetry of the Kerr Black Hole," Phys. Rev. D 82 (2010) 024008 [arXiv:1004.0996 [hep-th]].

[15] C. Krishnan, "Hidden Conformal Symmetries of Five-Dimensional Black Holes," JHEP 1007, 039 (2010) [arXiv:1004.3537 [hep-th]].

[16] C. M. Chen and J. R. Sun, "Hidden Conformal Symmetry of the Reissner-Nordström Black Holes," JHEP 1008 (2010) 034 [arXiv:1004.3963 [hep-th]].

[17] Y. Q. Wang and Y. X. Liu, "Hidden Conformal Symmetry of the Kerr-Newman Black Hole," JHEP 1008, 087 (2010) [arXiv:1004.4661 [hep-th]].

[18] B. Chen and J. Long, "Real-time Correlators and Hidden Conformal Symmetry in Kerr/CFT Correspondence," JHEP 1006, 018 (2010) [arXiv:1004.5039 [hep-th]].

[19] R. Li, M. F. Li and J. R. Ren, "Entropy of Kaluza-Klein Black Hole from Kerr/CFT Correspondence," Phys. Lett. B 691, 249 (2010) [arXiv:1004.5335 [hep-th]]. 
[20] D. Chen, P. Wang and H. Wu, "Hidden conformal symmetry of rotating charged black holes," [arXiv:1005.1404 [gr-qc]].

[21] M. Becker, S. Cremonini and W. Schulgin, "Correlation Functions and Hidden Conformal Symmetry of Kerr Black Holes," JHEP 1009, 022 (2010) [arXiv:1005.3571 [hep-th]].

[22] B. Chen and J. Long, "On Holographic description of the Kerr-Newman-AdS-dS black holes," JHEP 1008, 065 (2010) [arXiv:1006.0157 [hep-th]].

[23] H. Wang, D. Chen, B. Mu and H. Wu, "Hidden conformal symmetry of extreme and non-extreme Einstein-Maxwell-Dilaton-Axion black holes," JHEP 1011, 002 (2010) [arXiv:1006.0439 [gr-qc]].

[24] R. Fareghbal, "Hidden Conformal Symmetry of Warped $\mathrm{AdS}_{3}$ Black Holes," Phys. Lett. B 694, 138 (2010) [arXiv:1006.4034 [hep-th]].

[25] C. M. Chen, Y. M. Huang, J. R. Sun, M. F. Wu and S. J. Zou, "On Holographic Dual of the Dyonic Reissner-Nordstróm Black Hole," Phys. Rev. D 82 (2010) 066003 [arXiv:1006.4092 [hep-th]].

[26] C. M. Chen, Y. M. Huang, J. R. Sun, M. F. Wu and S. J. Zou, "Twofold Hidden Conformal Symmetries of the Kerr-Newman Black Hole," Phys. Rev. D 82 (2010) 066004 [arXiv:1006.4097 [hep-th]].

[27] R. Li, M. F. Li and J. R. Ren, "Hidden Conformal Symmetry of Self-Dual Warped $\mathrm{AdS}_{3}$ Black Holes in Topological Massive Gravity," [arXiv:1007.1357 [hep-th]].

[28] Y. Matsuo, T. Tsukioka and C. M. Yoo, "Notes on the Hidden Conformal Symmetry in the Near Horizon Geometry of the Kerr Black Hole," Nucl. Phys. B 844 (2011) 146 [arXiv:1007.3634 [hep-th]].

[29] B. Chen, J. Long and J. j. Zhang, "Hidden Conformal Symmetry of Extremal Black Holes," Phys. Rev. D 82 (2010) 104017 [arXiv:1007.4269 [hep-th]].

[30] K. N. Shao and Z. Zhang, "Hidden Conformal Symmetry of Rotating Black Hole with four Charges," [arXiv:1008.0585 [hep-th]].

[31] M. R. Setare and V. Kamali, "Hidden Conformal Symmetry of Rotating Black Holes in Minimal Five-Dimensional Gauged Supergravity," Phys. Rev. D 82, 086005 (2010) [arXiv:1008.1123 [hep-th]].

[32] A. M. Ghezelbash, V. Kamali and M. R. Setare, "Hidden Conformal Symmetry of Kerr-Bolt Spacetimes," [arXiv:1008.2189 [hep-th]].

[33] B. Chen and J. Long, "Hidden Conformal Symmetry and Quasi-normal Modes," [arXiv:1009.1010 [hep-th]].

[34] B. Chen, A. M. Ghezelbash, V. Kamali and M. R. Setare, "Holographic description of Kerr-Bolt-AdS-dS Spacetimes," [arXiv:1009.1497 [hep-th]].

[35] R. li and J. R. Ren, "Holographic Dual of Linear Dilaton Black Hole in Einstein-Maxwell-Dilaton-Axion Gravity," JHEP 1009, 039 (2010) [arXiv:1009.3139 [hep-th]].

[36] B. Chen, C. M. Chen and B. Ning, "Holographic Q-picture of Kerr-Newman-AdS-dS Black Hole," [arXiv:1010.1379 [hep-th]]. 
[37] M. R. Setare and V. Kamali, "Hidden Conformal Symmetry of Extremal Kerr-Bolt Spacetimes," JHEP 1010, 074 (2010) [arXiv:1011.0809 [hep-th]].

[38] C. M. Chen, V. Kamali and M. R. Setare, "Holographic Q-Picture of Black Holes in Five Dimensional Minimal Supergravity," [arXiv:1011.4556 [hep-th]].

[39] B. Chen and J. j. Zhang, "Quasi-normal Modes of Extremal Black Holes from Hidden Conformal Symmetry," [arXiv:1012.2219 [hep-th]].

[40] B. Chen and C. S. Chu, "Real-time correlators in Kerr/CFT correspondence," JHEP 1005 (2010) 004 [arXiv:1001.3208 [hep-th]].

[41] J. M. Maldacena and A. Strominger, "Universal low-energy dynamics for rotating black holes," Phys. Rev. D 56, 4975 (1997) [arXiv:hep-th/9702015].

[42] D. T. Son and A. O. Starinets, "Minkowski-space correlators in AdS/CFT correspondence: Recipe and applications," JHEP 0209, 042 (2002) [arXiv:hep-th/0205051].

[43] D. Rasheed, "The Rotating dyonic black holes of Kaluza-Klein theory," Nucl. Phys. B 454 (1995) 379 [arXiv:hep-th/9505038].

[44] F. Larsen, "Rotating Kaluza-Klein black holes," Nucl. Phys. B 575 (2000) 211 [arXiv:hep-th/9909102].

[45] T. Azeyanagi, N. Ogawa and S. Terashima, "Holographic Duals of Kaluza-Klein Black Holes," JHEP 0904, 061 (2009) [arXiv:0811.4177 [hep-th]].

[46] G. W. Gibbons and D. L. Wiltshire, "Black Holes In Kaluza-Klein Theory," Annals Phys. 167 (1986) 201 [Erratum-ibid. 176 (1987) 393].

[47] V. P. Frolov, A. I. Zelnikov and U. Bleyer, "Charged Rotating Black Hole From Five-Dimensional Point Of View," Annalen Phys. 44 (1987) 371.

[48] J. i. Koga and K. i. Maeda, "Evaporation and fate of dilatonic black holes," Phys. Rev. D 52 (1995) 7066 [arXiv:hep-th/9508029]. 